\title{
The FDA Nozzle Benchmark: In Theory There Is No Difference Between Theory and Practice, But in Practice There Is
}

\author{
A. W. Bergersen, M. Mortensen, and K. Valen-Sendstad*
}

\begin{abstract}
SUMMARY
The utility of flow simulations relies on the robustness of computational fluid dynamics (CFD) solvers and reproducibility of results. The aim of this study was to validate the Oasis CFD solver against in-vitro experimental measurements of jet breakdown location from the FDA nozzle benchmark at Reynolds number 3500 , which is in the particularly-challenging transitional regime. Simulations were performed on meshes consisting of 5, 10, 17 and 28 million (M) tetrahedra, with $\Delta t=10^{-5}$ seconds. The 5 and $10 \mathrm{M}$ simulation jets broke down in reasonable agreement with the experiments. However, the 17 and $28 \mathrm{M}$ simulation jets broke down further downstream. But which of our simulations are 'correct'? From a theoretical point of view, they are all wrong because the jet should not break down in the absence of disturbances. The geometry is axisymmetric with no geometrical features that can generate angular velocities. A stable flow was supported by linear stability analysis. From a physical point of view, a finite amount of 'noise' will always be present in experiments, which lowers transition point. To replicate noise numerically, we prescribed minor random angular velocities $(\sim 0.31 \%)$, much smaller than the reported flow asymmetry ( $\sim 3 \%)$ and model accuracy $(\sim 1 \%)$, at the inlet of the 17M simulation, which shifted the jet breakdown location closer to the measurements. Hence, the high-resolution simulations and 'noise' experiment can potentially explain discrepancies in transition between sometimes 'sterile' CFD and inherently noisy 'ground truth' experiments. Thus, we have shown that numerical simulations can agree with experiments, but for the wrong reasons. Copyright (C) 2018 John Wiley \& Sons, Ltd.
\end{abstract}

Received ...

KEY WORDS: Computational fluid dynamics; Turbulence; Transitional Flow; Validation; FDA nozzle benchmark; Noise

*Correspondence to: kvs@ simula.no 


\section{INTRODUCTION}

Cardiovascular diseases are burdening healthcare systems and costs are expected to rise in the years to come [1]. Systemic risk factors have been associated with higher prevalence of cardiovascular diseases, however, e.g., aneurysms [2] and atherosclerotic plaques [3] are focally distributed, highlighting the importance of blood-flow-induced wall shear stress [4, 5]. Medical image-based computational fluid dynamics (CFD) [6] has been extensively used retrospectively on large image databases to correlate abnormal stresses with disease initiation and outcome [7, 8], with the ultimate aim of using CFD as a prospective clinical tool. However, the efficacy of CFD depends on the robustness of the methods and reproducibility of results. The U.S. Food and Drug Administration (FDA) therefore devised a benchmark of a "generic medical device" [9] where the goal was to assess the state-of-the-art of CFD in biomechanics, comparing CFD solutions to in-vitro experiments, and to provide reference solutions for future validation of CFD solvers [10].

The inter laboratory comparison showed a relatively wide variability in the predicted breakdown location of the (possibly) turbulent jet [11]. Largest discrepancies were reported in the transitional flow regime, in contrast to the fully laminar and turbulent flows. Interestingly, none of the CFD benchmark participants obtained results in agreement with the in-vitro measurements for Reynolds numer (Re) 3500, although good agreement and excellent CFD results have been reported by multiple authors retrospectively $[12,13,14,15]$. The FDA nozzle benchmark model remains highly relevant for biomedical problems and our aim was to further validate the open-source CFD solver Oasis [16] that we have extensively used to study turbulent-like cardiovascular flows $[17,18,19,20$, $21,22,23]$. We focus on the flow at $R e=3500$, which is in the particularly-challenging transitional flow regime, for which the in-vitro experiments displayed the least variability.

\section{METHODOLOGY}

A sketch of the idealized medical device used in the FDA nozzle benchmark is shown in Figure 1. The inlet pipe in the in-vitro experimental setup was $2.661 \mathrm{~m}$ long with an outlet section of 1.146 $\mathrm{m}$. The computational domain was chosen to be shorter than the in-vitro one, in total $0.320 \mathrm{~m}$ long, from $z=-0.120 \mathrm{~m}$ to $z=0.200 \mathrm{~m}$ relative to the sudden expansion located at $z=0 \mathrm{~m}$ $(x, y=0 \mathrm{~m})$. To ease reproducibility of our results, unstructured volumetric meshes were created with constant node spacing and four boundary layers using ICEM-CFD (ANSYS Inc., Canonsburg, PA, USA). In total 4 meshes where created consisting of 5, 10, 17 and 28 million (M) tetrahedron cells, referred to as $5 \mathrm{M}, 10 \mathrm{M}, 17 \mathrm{M}$, and $28 \mathrm{M}$, respectively. The characteristic node spacing for these meshes were $3.5 \cdot 10^{-4}, 2.8 \cdot 10^{-4}, 2.4 \cdot 10^{-4}$, and $2.0 \cdot 10^{-4} \mathrm{~m}$. We specified a constant time step of $\Delta t=1 \cdot 10^{-5}$ seconds for all simulations, based on setting the maximum Courant number to 0.5 , assuming a peak centerline velocity of $4 \mathrm{~m} / \mathrm{s}$ and using the minimum cell length of the $28 \mathrm{M}$ mesh. The initial condition was set to zero for both velocity and pressure, and we specified a parabolic velocity profile at the inlet. The pressure was set to zero at the outlet and we applied a no-slip condition at the walls. Simulations were performed using Oasis, where special care has been taken to ensure a kinetic-energy-preserving and minimally-dissipative numerical solution. The solver and numerical implementation is described in detail elsewhere [16]. The instantaneous velocity, $\boldsymbol{u}(\boldsymbol{x}, t)$, was sampled at various points and cross sections along the $z$-axis, including at $z=-0.016 \mathrm{~m}, 0 \mathrm{~m}$, $0.04 \mathrm{~m}, 0.08 \mathrm{~m}, 0.12 \mathrm{~m}$, and $0.16 \mathrm{~m}$. Reynolds decomposition was used to separate the instantaneous velocity from the time averaged, $\overline{\boldsymbol{u}}(\boldsymbol{x})$, and the fluctuating, $\boldsymbol{u}^{\prime}(\boldsymbol{x}, t)$, components, i.e., $\boldsymbol{u}=\overline{\boldsymbol{u}}+\boldsymbol{u}^{\prime}$. We also computed the turbulent kinetic energy (TKE) as $k=\frac{1}{2}\left(\boldsymbol{u}^{\prime} \cdot \boldsymbol{u}^{\prime}\right)$, and power spectral density (PSD) of the fluctuating velocity magnitude, $\left|\boldsymbol{u}^{\prime}\right|$, at various locations along the centerline. For the latter, we used Welch's method [24] with 8 segments, and a Hanning windowing function with 50\% overlap. 


\section{RESULTS}

We focus first on time-averaged cross-sectional and centerline velocities sampled between $t=$ $1.4-1.6$ second, when the jet breakdown locations had stabilized, shown in Figure 2, left and right, respectively. Relative to the $5 \mathrm{M}$ simulation, the $10 \mathrm{M}$ simulation appears to converge towards the in-vitro measurements. However, the $17 \mathrm{M}$ and $28 \mathrm{M}$ simulations broke down $\sim 10$ and 15 inlet diameters further downstream.

To investigate the apparent discrepancies in time-averaged jet breakdown location, Figure 3 (top) shows the instantaneous velocity fields for the four different mesh densities at $t=1.0$ second. Figure 3 (bottom) shows the instantaneous velocity magnitude, TKE, and PSD of the fluctuating velocity component for $t=1.0-1.1$ seconds in subplots $\mathrm{a}, \mathrm{b}$, and $\mathrm{c}$, respectively, where the prefix corresponds to the probe locations in Figure 3 (top). Focusing now on subplots 1a and 1b, just upstream of the sudden expansion, there are no apparent velocity fluctuations. In contrast, at the sudden expansion, the fluctuations are clearly visible in plot $2 \mathrm{a}$ and $2 \mathrm{~b}$ for the 5 and $10 \mathrm{M}$ simulations. This is also reflected by $2 \mathrm{c}$, displaying fluctuations that contained additional energy, compared to $1 \mathrm{c}$. However, the 17 and $28 \mathrm{M}$ simulations only contained low energy and low frequency flow instabilities. Further downstream, in plot 3 a, the centerline velocity magnitude is reduced for the 5 and $10 \mathrm{M}$ simulations, and the $\left|\boldsymbol{u}^{\prime}\right|$ has more energy in the higher frequencies in $3 \mathrm{~b}$, also reflected by $3 \mathrm{c}$. On the other hand, the 17 and $28 \mathrm{M}$ simulations are at location 3 practically identical to location 2. Further downstream, in plots $4 \mathrm{a}$ and $4 \mathrm{~b}$, both the 5 and $10 \mathrm{M}$ simulations developed similarly; the centerline velocity was relatively stable and only contained low amplitude and low frequency fluctuations. Subplot $4 \mathrm{c}$ reveals that flow instabilities in the 17 and $28 \mathrm{M}$ simulations grew, whereas the $5 \mathrm{M}$ and $10 \mathrm{M}$ flows were further dissipated. Approximately 10 diameters downstream of the sudden expansion, the $17 \mathrm{M}$ jet broke down as reflected by plots 5a-c. The energy spectra in plot $5 \mathrm{c}$ shows that the $28 \mathrm{M}$ simulation instabilities increased relative to location 4 . In the last column of Figure 3 we can see that the centerline velocity of $5 \mathrm{M}, 10 \mathrm{M}$, and $17 \mathrm{M}$ had close to the same magnitude, although $17 \mathrm{M}$ exhibit larger fluctuations. Furthermore, the jet in the $28 \mathrm{M}$ simulation had at this location broken down, as shown in plot 6a-c, whereas the flow in the 17M simulation had lost much of its high frequency components, as reflected by the PSD, indicative of further flow stabilization.

\section{DISCUSSION}

The aim of this study was to validate the CFD solver Oasis against the in-vitro measurements presented in the FDA nozzle benchmark. However, our results would seem to suggest that we have refuted the validity of our solver instead. But, before concluding that our solver is erroneous, let us consider a few aspects of the FDA nozzle benchmark and our approach. From a purely computational point of view, we would intuitively put more faith in the more resolved simulations, but the 5 and $10 \mathrm{M}$ simulation results were closer to the in-vitro experimental measurements. This apparent contradiction led to the obvious question; which , if any, of our simulations are 'correct'?

The consistent correlation between increased mesh resolution and jet breakdown location reminded us that the geometry is fully axisymmetric, so there should not be any 3D structures as observed in the abrupt jet breakdown. Said in other words, all our results are actually wrong. From a purely theoretical point of view, it is established that fully axisymmetric flows are known to not transition to turbulence, because there are no geometrical features that can introduce asymmetries. This is conceptually easy to comprehend by rewriting the Navier-Stokes equations into cylindrical coordinates. With the prescribed boundary conditions, the solution becomes independent of the angular direction and thereby just a collection of identical 2D planes. This has also been shown computationally, that fully axisymmetric flows only break down to turbulence if the numerical solution is perturbed, e.g., numerically [25] or geometrically [26, 27].

To investigate our results from an analytical point of view we used linear stability analysis. In short, we decomposed the flow variables into the sum of a stable laminar base flow and a perturbation $(\boldsymbol{u}, p)=(\boldsymbol{U}, P)+\left(\boldsymbol{u}^{\prime}, p^{\prime}\right)$. The decomposition was inserted into the Navier-Stokes 
equations that were linearized after eliminating the pressure using the continuity condition [28]. We then obtained a linear operator equation for the evolution of the velocity perturbation

$$
\frac{\partial \boldsymbol{u}^{\prime}}{\partial t}=L\left(\boldsymbol{u}^{\prime}\right)
$$

where $L$ is the linearized operator. The eigensystem of $L$ is given by the eigenvalues $\lambda_{\alpha}$ and eigenmodes $\tilde{\boldsymbol{u}}_{\alpha}^{\prime}$ as $L \tilde{\boldsymbol{u}}_{\alpha}^{\prime}=\lambda_{\alpha} \tilde{\boldsymbol{u}}_{\alpha}^{\prime}$ (no summation on $\alpha$ ). Given the eigensystem, the perturbed velocity vector may be obtained as

$$
\boldsymbol{u}^{\prime}=\sum_{\alpha=0}^{\infty} \exp \left(\lambda_{\alpha} t\right) \tilde{\boldsymbol{u}}_{\alpha}^{\prime} .
$$

A linear stability analysis amounts to computing the leading eigenvalues of the linearized NavierStokes operator $L$, as well as the corresponding eigenmodes $\tilde{\boldsymbol{u}}_{\alpha}^{\prime}$, representing the perturbations to the laminar base flow. The growth rate of the perturbations (i.e., the eigenvalues) are indicative of whether or not the flow is linearly stable; a negative eigenvalue represents a stable mode and a positive eigenvalue represents an unstable mode. If perturbing a numerical simulation with an unstable mode and allowed to grow sufficiently in time, the eigenmode will eventually trigger turbulence in numerical simulations [25].

It is evident that the flow regime upstream of the sudden expansion $(\mathrm{z}=0)$ will have a profound effect on the flow in the main pipe and jet breakdown location. We therefore split the domain in Figure 1 at $z=0 \mathrm{~m}$, and computed two analyses; one upstream and one downstream of the sudden expansion, using the open source spectral element code Semtex [29] together with its accompanying Dog [28] for the linear stability analyses.

The analyses revealed that all eigenvalues for the flow in the upstream section were negative, indicative of a stable and laminar flow. In contrast, the outlet section contained positive eigenvalues and thus unstable eigenmodes, which are visualized in Figure 4. Of note from the analyses is: First, the flow in the throat section should be laminar, and second linear stability analysis supports the experimental observations that potential instabilities should grow and cause jet breakdown. The predicted laminar flow in the throat section is of utmost importance to explain our numerical results, since flow instabilities at the sudden expansion dictate jet breakdown location. That being said, linear stability theory cannot provide definite proof and is often conservative compared to invitro experiments, not to mention the unphysical prediction of laminar pipe flow even at infinite Re [30]. Alternatively, quoting Carstensen et al. [31], when comparing experimental values for transitional and critical Reynolds number obtained through theoretical stability analysis (i.e. when linear disturbances start to grow), any correspondence in the values is usually just a coincidence.

Since linear stability analysis predicted a laminar and stable flow, our natural follow-up question was 'what caused asymmetrical flow components in our simulations'? To understand our results from a numerical point of view, the first clue was found in subplot $1 \mathrm{c}$ of Figure 3 . We observed that the two coarsest simulations exhibit low amplitude, but high frequency, 'noise' in the $\sim 0-3000 \mathrm{~Hz}$ range, upstream of the sudden expansion, that were absent in the two finer ones. It is rather intuitive that the Cartesian tetrahedral mesh is the source of the noise, as all other simulation parameters were kept fixed. By approximating a cylindrical geometry with an increasing number of linear elements, the geometry is more accurately represented, and the numerical accuracy is improved. The former is rather intuitive and the latter is elementary knowledge [32], but the mesh quality is generally also improved.

To isolate and investigate the effect of mesh quality on numerical accuracy, and hence accurately predict transitional flows, we compared numerical solutions on meshes with optimal versus suboptimal aspect ratio, respectively. We chose the 3D Taylor-Green vortex benchmark [33] where the boundary conditions are periodic on the domain $\Omega \in[-2 \pi, 2 \pi]^{3}$, which makes the problem conceptually unbounded and independent of geometrical features. The initial conditions are analytical vortices [34] that break down to consecutively smaller ones until dissipation dominates at the smaller scales. The evolution of the vortices is symmetrical and deterministic, but the flow has an energy cascade and other features commonly associated with truly turbulent flows [35]. We 
set $R e=1000, \Delta t=1 \cdot 10^{-3}$ seconds, and computed the solution on meshes consisting of $6 \cdot 32^{3}$, $6 \cdot 64^{3}$, and $6 \cdot 128^{3}$ tetrahedral cells, where the interior node locations were perturbed by a vector drawn from a normal distribution with standard deviation of $18 \%$ and $36 \%$ of the tetrahedron node spacing, respectively, reflecting mesh quality observed in the 28 and $10 \mathrm{M}$ element meshes.

The results of our simple and controlled numerical experiment is presented in Figure 5, showing temporal evolution of the rate of dissipation, $\epsilon=-\partial k / \partial t$. The solutions on the three unperturbed meshes are shown in Figure 5 a), where we can observe that the numerical solutions evolves equally up until $t \sim 3$ seconds on all meshes. However, after $t \geq 3$ seconds the coarse mesh resolution leads to a premature elevation of $\epsilon$, as vortices breaking down below the mesh resolution aren't dissipated. Figures 5 b-d) shows a systematic shift in point of transition caused by flow asymmetries introduced by the perturbed node locations. These effects are more pronounced on the coarse meshes that are under-resolved and the flow reaches a complex and chaotic state sooner. We can also observe that distorted elements have less of an effect on the better resolved mesh, as, e.g., 18\% distortion is equivalent to $36 \%$ on the $6 \cdot 64^{3}$ versus $6 \cdot 128^{3}$ cell mesh, respectively. We here observe in isolation the same effects as in our FDA nozzle benchmark results, namely that transition occurs earlier on the lower quality and coarser meshes, but here in the absence of geometrical features.

From a physical point of view, it is difficult to completely exclude minor imperfections in invitro laboratory experiments, stemming from either minor pulsations in the flow rate caused by a pump, deflected pipes, transitions between pipes, minor surface irregularities in the geometry etc., here collectively referred to as experimental noise. That experimental noise might affect the critical $R e$, i.e., for which $R e$ the flow deviates from a laminar regime, was reported by Reynolds already in 1883 [36]. Reynolds observed a turbulent flow regime down to $R e \sim 2000$, but also laminar flow up to $R e \sim 13000$; solely dependent on the level of experimental noise. On the high side, conceptually similar in-vitro experiments have shown laminar pipe flow for $R e$ up to 100000 by taking extreme care to reduce asymmetries and noise [37]. On the low side, flow instabilities due to asymmetries as small as model manufacturing precision has been studied in both idealized [38, 39] and patient-specific [40] stenosed artery models. For comparison, the physical model uncertainty in the FDA nozzle benchmark was reported to be within $1 \%$, flow rate fluctuation less than $1 \%$, and that the particle-image-velocimetry-measured time-averaged flow asymmetry was within 3\% [10] at the entrance of the nozzle for $R e=3500$. Furthermore, using laser Doppler velocimetry with the same experimental set-up and standard operating procedure as in [10], Taylor et al. reported that small perturbations were present in the throat section in-vitro at $R e=2000$ [41].

As noted in the previous paragraph, determining the point of transition can be very challenging. Therefore, the in-vitro results are excellent from an experimental point of view, with good interlaberatory agreement. However, experiments intended for validation of numerical solvers have to provide measured, not idealized, boundary conditions of the experiment to ensure 'numerical reproducibility' [42]. In the context of the FDA nozzle benchmark, modelers might not have been provided with precise enough information to replicate the observed deviations from a laminar profile, and therefore simulated an idealized version of the experiment instead, which might not have occurred experimentally. It is fully possible that the latter can explain the observed differences between the in-vitro and in-silico results, also supported by [15].

One way to numerically mimic potential experimental noise is to perturb the numerical simulation with a finite level of noise to break the aforementioned symmetry. We therefore added white noise at the inlet of the $17 \mathrm{M}$ element mesh, which was the computationally least expensive mesh where the simulation results showed discrepancies with in-vitro measurements. More specifically, we prescribed random velocity components in the angular direction only, drawn from a normal distribution with mean of zero, and a standard deviation of $0.001 \mathrm{~m} / \mathrm{s}$. Relative to the FDA nozzle benchmark, this standard deviation was $0.31 \%$ of the cross-sectional mean axial inlet velocity at $R e=3500$, and one order of magnitude less than the experimentally measured left/right timeaveraged flow asymmetry. The interesting feature is whether this noise decays or grows, i.e., if the noise is over or under the critical level of noise [43]. We therefore performed simulations of flows in the laminar $(R e=500)$, transitional $(R e=3500)$, and turbulent $(R e=6500)$ regimes, both with and without noise, referred to as noise and no-noise, respectively. 
Figure 6 shows the time-averaged centerline velocities for $R e=500,3500$, and 6500 , with noise and no-noise compared against the in-vitro experiment measurements [10]. We observe that both the $R e=500$ and $R e=6500$ flow simulations were unaffected by noise, as the noise/no-noise results are indistinguishable and show excellent agreement with the experiments. On the other hand, the $R e=3500$ flow results were 'strongly' affected by noise, as the jet breakdown location shifted $\sim 9$ diameters upstream. Comparing against in-vitro and in-silico pipe flow experiments, the changes in $R e=3500$ simulation were expected, since critical threshold of noise typically scales with $\frac{1}{R e}$ [44]. Therefore, neither of the 'extreme' Re simulations should be affected by noise, only those in the transitional regime. Admittedly, the $R e=3500$ simulation with noise did not show a perfect agreement with the in-vitro measurements. In contrast to the laboratory experiments, the random noise we added did not a priori satisfy the Navier-Stokes equations, and was only introduced at one location. Furthermore, the noise was largely dissipated before reaching the sudden expansion for $R e=3500$, consistent with the linear stability analysis results. However, this non-exhaustive ad-hoc numerical experiment was only intended as a proof-of-concept that noise might lower the critical Reynolds number for transitional flows. Further investigation of how different types of noise can affect transition is a scientifically important and interesting topic, but beyond the scope of this study, cf., [44, 45].

Quoting Oberkampf and Roy, 'knowing the correct answer beforehand is extremely seductive, even to a saint' [42]. In this context, and relative to other groups, we are aware of a handful of studies where the authors, like us, had access to the in-vitro 'ground-truth' experimental results prior to simulating the flows, which allows for tweaking and tuning of parameters to match the experiments. Solution strategies include direct numerical simulation [12], large eddy simulation (LES) [13, 14, 15], or a dynamic hybrid Reynolds-averaged Navier-Stokes (RANS)/LES model [46]. The fact that RANS [47] and LES [48] models are generally too dissipative, and not suitable to studying flows in the transitional regime, is beyond the point; all studies where the authors were non-blinded to the in-vitro results showed reasonable agreement with the measurements.

Passerini et al. [12] used a locally refined mesh with $\sim 3 \mathrm{M}$ cells and quadratic Taylor-Hood elements $\left(\mathbb{P}_{2}-\mathbb{P}_{1}\right)$, which is equivalent of $24 \mathrm{M}$ linear $\left(\mathbb{P}_{1}-\mathbb{P}_{1}\right)$ elements. Passerini et al. assessed the spatially varying relative mesh resolution computing $l^{+}[49]$ and reported $l_{\max }^{+}=4$, indicative of a spatially well-resolved simulation. We also ran our $10 \mathrm{M}$ element simulation using quadratic Taylor-Hood elements $\left(\mathbb{P}_{2}-\mathbb{P}_{1}\right)$, which is equivalent of $80 \mathrm{M}$ linear $\left(\mathbb{P}_{1}-\mathbb{P}_{1}\right)$ elements at a time step size of $\Delta t=5 \cdot 10^{-6}$ seconds [50]. The jet breakdown location shifted continuously downstream of the sudden expansion and eventually reached the end of the computational domain, which caused backflow at the outlet and a diverged numerical solution. The latter is wellknown $[40,51]$, however Passerini et al. still reported a lower $l_{\max }^{+}$compared to our $l_{\max }^{+}=$ $11.0\left(l_{\text {average }}^{+}=1.3\right)$ obtained on the $80 \mathrm{M}$ linear $\left(\mathbb{P}_{1}-\mathbb{P}_{1}\right)$ element equivalent mesh with constant node spacing. This comparison may indicate that the mesh used by Passerini et al. was rather refined in the high shear rate regions, and consequently equally coarser elsewhere. This might suggest that numerical noise might have been introduced by a locally coarse mesh, which resulted in overall good agreement with the experimental measurements. That being said, a head-to-head comparison is not easy due to mesh reproducibility issues.

Delorme et al. [14] performed a refinement study for $R e=2000$, where the most resolved mesh had the equivalent to $\sim 42 \mathrm{M}$ linear elements. Although the mesh resolution does not differ much from ours, the results still do. That being said, it is difficult searching for meaningful sources of discrepancies as they used a structured staggered mesh, the finite volume method, and an LES model, all different from our modeling choices.

Zmijanovic et al. [15] made an excellent point assessing the numerical robustness of the nozzle benchmark by investigating the sensitivity of spatial and temporal resolution, temporal discretization schemes, and turbulent intensity (TI) injection on jet breakdown location. Regarding the latter, TI injection was found to produce robust numerical results relative to the experiments, regardless of intensity. Focusing now exclusively on simulations without TI injection, Zmijanovic et al. also compared the jet breakdown location between an explicit fourth order Runge-Kutta (RK4) scheme, and a linear combination of a two-step time-explicit Taylor-Galerkin scheme (20\%) and 
the RK4 scheme (80\%), referred to as TFV4A, for various time step sizes with a $5 \mathrm{M}$ and $15 \mathrm{M}$ element mesh. When improving the temporal resolution they reported no or a downstream shift for RK4, versus an upstream shift for TFV4A, relative to the in-vitro experiments. In their spatial refinement study Zmijanovic et al. reported an excellent agreement with the in-vitro experiments for the 50M-element simulation, whereas an up- and downstream shift for the 5 and $15 \mathrm{M}$-element simulations, respectively using the TFV4A scheme. In contrast to our results, Zmijanovic et al. report an inconsistent effect of the spatial and temporal resolution on jet breakdown location, and it is therefore unclear what breaks the axis symmetry in their simulations without TI injection. That being said, our studies are largely complimentary, both investigating the impact of numerical noise and solver settings on jet breakdown location.

A teaching moment from the current study may be that our high resolution simulations and numerical noise experiments can potentially shed light on the observed discrepancies in transition to turbulence between sometimes 'sterile' CFD and 'ground truth' in-vitro experiments that are inherently 'noisy' [52]. Secondly, although symmetric models are convenient to manufacture, warnings about the use of such has been put forward as one is literally 'dancing on the knife-edge of symmetry' [53]. Finally, our original aim was to validate our solver against in-vitro measurements. As shown in Figure 6, we demonstrate excellent agreement with the measurement for the fully laminar and turbulent flow regimes. On the other hand, the transitional regime is surprisingly sensitive to minor perturbations, as discussed above. However, having provided new insight into the source of numerical noise, we would still not refute the validity of our solver in the transitional regime

\section{CONCLUSIONS}

We have performed CFD simulations of the FDA nozzle benchmark for various Reynolds numbers and mesh resolutions. The coarse simulation results showed an overall acceptable agreement with the experimental measurements, whereas the finer ones broke down much further downstream. The discrepancies were attributed to numerical noise introduced by mesh artifacts, which were more profound in the coarse meshes. We conclude that the jet in the FDA nozzle benchmark should in the absence of disturbances not transition to turbulence from a theoretical point of view, although from a practical point of view, the jet breaks down both in-vitro and in-silico, depending on the type and level of noise. We can conclude that the onset of transition remains challenging to predict, including how mesh artifacts affects the critical $R e$ in simulations. Hence, our results can potentially shed light on the observed discrepancies in transition between CFD that can be sterile and in-vitro 'ground truth' experiments that are inherently noisy, or that 'in theory there is no difference between theory and practice, but in practice there is' [54]. In other words, we have shown that numerical simulation results can agree with experiments, but for the wrong reasons.

\section{ACKNOWLEDGEMENTS}

The study was supported by The Research Council of Norway through a Centres of Excellence grant to the Center for Biomedical Computing at Simula Research Laboratory, project number 179578. This work was also carried out as a part of the Centre for Cardiological Innovation, and SIMMIS, project number 262827, funded by the Research Council of Norway. Simulations were performed on the Abel Cluster, owned by the University of Oslo and the Norwegian metacenter for High Performance Computing (NOTUR), and operated by the Department for Research Computing at USIT, the University of Oslo IT-department, grant number nn9316k. 


\section{REFERENCES}

\section{REFERENCES}

1. Murray CJ, Lopez AD. Alternative projections of mortality and disability by cause 1990-2020: Global burden of disease study. The Lancet 1997; 349(9064):1498 - 1504.

2. Weir B. Unruptured intracranial aneurysms: A review. Journal of Neurosurgery 2002; 96(1):3-42.

3. Lee SW, Antiga L, Spence JD, Steinman DA. Geometry of the carotid bifurcation predicts its exposure to disturbed flow. Stroke 2008; 39(8):2341-2347.

4. Malek AM, Alper SL, Izumo S. Hemodynamic shear stress and its role in atherosclerosis. JAMA 1999; 282(21):2035-2042.

5. Morbiducci U, Kok AM, Kwak BR, Stone PH, Steinman DA, Wentzel JJ, et al.. Atherosclerosis at arterial bifurcations: Evidence for the role of haemodynamics and geometry. Thrombosis and Haemostasis 2016; 115(3):484-492.

6. Taylor CA, Steinman DA. Image-based modeling of blood flow and vessel wall dynamics: Applications, methods and future directions. Annals of Biomedical Engineering Mar 2010; 38(3):1188-203.

7. Chen H, Selimovic A, Thompson H, Chiarini A, Penrose J, Ventikos Y, Watton PN. Investigating the influence of haemodynamic stimuli on intracranial aneurysm inception. Annals of Biomedical Engineering Apr 2013; .

8. Cebral JR, Raschi M. Suggested connections between risk factors of intracranial aneurysms: A review. Annals of Biomedical Engineering 2013; 41(7):1366-1383.

9. Website. U.S. Food and Drug Administration. https://fdacfd.nci.nih.gov. Accessed June 20, 2018.

10. Hariharan P, Giarra M, Reddy V, Day SW, Manning KB, Deutsch S, Stewart SF, Myers MR, Berman MR, Burgreen GW, et al. Multilaboratory particle image velocimetry analysis of the FDA benchmark nozzle model to support validation of computational fluid dynamics simulations. Journal of Biomechanical Engineering 2011; 133(4):041 002.

11. Stewart SF, Paterson EG, Burgreen GW, Hariharan P, Giarra M, Reddy V, Day SW, Manning KB, Deutsch S, Berman MR, et al. Assessment of CFD performance in simulations of an idealized medical device: Results of FDAs first computational interlaboratory study. Cardiovascular Engineering and Technology 2012; 3(2):139-160.

12. Passerini T, Quaini A, Villa U, Veneziani A, Canic S. Validation of an open source framework for the simulation of blood flow in rigid and deformable vessels. International Journal for Numerical Methods in Biomedical Engineering 2013; 29(11):1192-1213.

13. Janiga G. Large eddy simulation of the FDA benchmark nozzle for a Reynolds number of 6500. Computers in Biology and Medicine 2014; 47:113-119.

14. Delorme YT, Anupindi K, Frankel SH. Large eddy simulation of FDAs idealized medical device. Cardiovascular Engineering and Technology 2013; 4(4):392-407.

15. Zmijanovic V, Mendez S, Moureau V, Nicoud F. About the numerical robustness of biomedical benchmark cases: Interlaboratory FDA's idealized medical device. International Journal for Numerical Methods in Biomedical Engineering 2017; 33(1).

16. Mortensen M, Valen-Sendstad K. Oasis: A high-level/high-performance open source Navier-Stokes solver. Computer Physics Communications 2015; 188:177-188.

17. Valen-Sendstad K, Mardal KA, Steinman DA. High-resolution CFD detects high-frequency velocity fluctuations in bifurcation, but not sidewall, aneurysms. Journal of Biomechanics 2013; 46(2):402-407.

18. Valen-Sendstad K, Steinman DA. Mind the gap: Impact of computational fluid dynamics solution strategy on prediction of intracranial aneurysm hemodynamics and rupture status indicators. American Journal of Neuroradiology 2014; 35(3):536-543.

19. Valen-Sendstad K, Piccinelli M, Steinman DA. High-resolution computational fluid dynamics detects flow instabilities in the carotid siphon: Implications for aneurysm initiation and rupture? Journal of Biomechanics 2014; 47(12):3210-3216.

20. Khan M, Valen-Sendstad K, Steinman D. Narrowing the expertise gap for predicting intracranial aneurysm hemodynamics: Impact of solver numerics versus mesh and time-step resolution. American Journal of Neuroradiology 2015; 36(7):1310-1316.

21. Mynard JP, Valen-Sendstad K. A unified method for estimating pressure losses at vascular junctions. International Journal for Numerical Methods in Biomedical Engineering 2015; 31(7).

22. Khan M, Steinman D, Valen-Sendstad K. Non-Newtonian versus numerical rheology: Practical impact of shearthinning on the prediction of stable and unstable flows in intracranial aneurysms. International Journal for Numerical Methods in Biomedical Engineering 2017; 33(7).

23. Khan MO, Chnafa C, Gallo D, Molinari F, Morbiducci U, Steinman DA, Valen-Sendstad K. On the quantification and visualization of transient periodic instabilities in pulsatile flows. Journal of Biomechanics 2017; 52:179-182.

24. Welch P. The use of fast Fourier transform for the estimation of power spectra: A method based on time averaging over short, modified periodograms. IEEE Transactions on Audio and Electroacoustics 1967; :70-73.

25. Sherwin S, Blackburn HM. Three-dimensional instabilities and transition of steady and pulsatile axisymmetric stenotic flows. Journal of Fluid Mechanics 2005; 533:297-327.

26. Varghese SS, Frankel SH, Fischer PF. Direct numerical simulation of stenotic flows. Part 1. Steady flow. Journal of Fluid Mechanics 7 2007; 582:253-280.

27. Varghese SS, Frankel SH, Fischer PF. Direct numerical simulation of stenotic flows. Part 2. Pulsatile flow. Journal of Fluid Mechanics 7 2007; 582:281-318.

28. Blackburn H. Working Dog. URL http://users.monash.edu.au/ bburn/pdf/guidedog.pdf, Accessed: June 20, 2018. 
29. Blackburn HM, Sherwin S. Formulation of a Galerkin spectral element-Fourier method for three-dimensional incompressible flows in cylindrical geometries. Journal of Computational Physics 2004; 197(2):759-778.

30. Drazin PG, Reid WH. Hydrodynamic stability. Cambridge university press, 2004.

31. Carstensen S, Sumer BM, Fredsøe J. Coherent structures in wave boundary layers. Part 1. Oscillatory motion. Journal of Fluid Mechanics 2010; 646:169-206.

32. Brenner S, Scott R. The mathematical theory of finite element methods, vol. 15. Springer Science \& Business Media, 2007.

33. Brachet ME, Meiron DI, Orszag SA, Nickel B, Morf RH, Frisch U. Small-scale structure of the Taylor-Green vortex. Journal of Fluid Mechanics 1983; 130:411-452.

34. Taylor G, Green A. Mechanism of the production of small eddies from large ones. Proceedings of the Royal Society of London. Series A, Mathematical and Physical Sciences 1937; 158(895):499-521.

35. Brachet M. Direct simulation of three-dimensional turbulence in the Taylor-Green vortex. Fluid Dynamics Research $1991 ; 8(1): 1-8$

36. Reynolds O. An experimental investigation of the circumstances which determine whether the motion of water shall be direct or sinuous, and of the law of resistance in parallel channels. Proceedings of the Royal Society of London $1883 ;$ 35(224-226):84-99.

37. Pfenninger W. Boundary layer suction experiments with laminar flow at high Reynolds numbers in the inlet length of a tube by various suction methods. Boundary Layer and Flow Control, Lachmann G (ed.). Pergamon, 1961; 961 -980 .

38. Cassanova R, Giddens D. Disorder distal to modeled stenoses in steady and pulsatile flow. Journal of Biomechanics 1978; 11(10-12):441-453.

39. Vétel J, Garon A, Pelletier D, Farinas MI. Asymmetry and transition to turbulence in a smooth axisymmetric constriction. Journal of Fluid Mechanics 2008; 607:351-386.

40. Fischer PF, Loth F, Lee SE, Lee SW, Smith DS, Bassiouny HS. Simulation of high-Reynolds number vascular flows. Computer Methods in Applied Mechanics and Engineering 2007; 196(31):3049-3060.

41. Taylor JO, Good BC, Paterno AV, Hariharan P, Deutsch S, Malinauskas RA, Manning KB. Analysis of transitional and turbulent flow through the FDA benchmark nozzle model using laser Doppler velocimetry. Cardiovascular Engineering and Technology 2016; 7(3):191-209.

42. Oberkampf WL, Roy CJ. Verification and validation in scientific computing. Cambridge University Press, 2010

43. Mullin T. Experimental studies of transition to turbulence in a pipe. Annual Review of Fluid Mechanics 2011; 43:1-24.

44. Hof B, Juel A, Mullin T. Scaling of the turbulence transition threshold in a pipe. Physical review letters 2003; 91(24):244 502.

45. Peixinho J, Mullin T. Finite-amplitude thresholds for transition in pipe flow. Journal of Fluid Mechanics 2007; 582:169-178.

46. Bhushan S, Walters DK, Burgreen GW. Laminar, turbulent, and transitional simulations in benchmark cases with cardiovascular device features. Cardiovascular Engineering and Technology 2013; 4(4):408-426.

47. Varghese SS, Frankel SH. Numerical modeling of pulsatile turbulent flow in stenotic vessels. Journal of Biomechanical Engineering 2003; 125(4):445-460.

48. Ducros F, Comte P, Lesieur M. Large-eddy simulation of transition to turbulence in a boundary layer developing spatially over a flat plate. Journal of Fluid Mechanics 1996; 326:1-36.

49. Valen-Sendstad K, Mardal KA, Mortensen M, Reif BAP, Langtangen HP. Direct numerical simulation of transitional flow in a patient-specific intracranial aneurysm. Journal of biomechanics 2011; 44(16):2826-2832.

50. Bergersen AW. Investigating the link between patient-specific morphology and hemodynamics: Implications for aneurysm initiation? 2016. URL https://www. duo.uio.no/bitstream/handle/10852/50515/ master-bergersen.pdf, Masters Thesis, University of Oslo, Norway.

51. Bertoglio C, Caiazzo A, Bazilevs Y, Braack M, Esmaily M, Gravemeier V, L Marsden A, Pironneau O, E VignonClementel I, A Wall W. Benchmark problems for numerical treatment of backflow at open boundaries. International journal for numerical methods in biomedical engineering 2018; 34(2):e2918.

52. Griffith MD, Leweke T, Thompson MC, Hourigan K. Effect of small asymmetries on axisymmetric stenotic flow. Journal of Fluid Mechanics 2013; 721:R1.

53. Steinman DA, Kehoe SC, Ford MD, Nikolov HN, Holdsworth DW. Dancing on the knife-edge of symmetry: On the misuse of symmetric models for studying blood flow dynamics. Proceedings of ASME 2003 Summer Bioengineering Conference, 2003; 25-29.

54. Ordsmith R. Pascal-an introduction to the art and science of programming. University Computing 1988; 10(3):164164. 